\title{
Allyl isothiocyanate increases MRP1 expression in cigarette smoke extract-stimulated human bronchial epithelial cells via the JNK/Nrf2 pathway
}

\author{
MIN ZHANG $^{1 *}$, SHUJUN WANG ${ }^{2 *}$, XUEQI WANG ${ }^{1}$, XIAOYA XU $^{1}$, ZHAOMIN YAO $^{1}$, \\ WEI FANG ${ }^{1}$, JIE WU $^{1}$, QINGQING WU ${ }^{1}$, ZEGENG LI $^{3}$ and DIANLEI WANG ${ }^{1,4}$ \\ ${ }^{1}$ School of Pharmacy, Anhui University of Chinese Medicine, Hefei, Anhui 230012; ${ }^{2}$ School of Pharmacy, \\ Zhejiang Pharmaceutical College, Ningbo, Zhejiang 315100; ${ }^{3}$ Department of Respiratory Medicine, \\ The First Affiliated Hospital to Anhui University of Chinese Medicine, Hefei, Anhui 230031; \\ ${ }^{4}$ Anhui Province Key Laboratory of Chinese Medicinal Formula, Anhui University of \\ Chinese Medicine, Hefei, Anhui 230012, P.R. China
}

Received October 25, 2019; Accepted January 14, 2021

DOI: $10.3892 /$ etm.2021.9840

\begin{abstract}
Multidrug resistance-related protein 1 (MRP1) is involved in the biological transport of several molecules with diverse structural characteristics outside of the cell. In addition to its transport activity, MRP1 exhibits multiple defense mechanisms in vivo. MRP1 is highly expressed in normal lung tissues and plays a protective role in the process of chronic obstructive pulmonary disease. In the present study, human bronchial epithelial cells (16HBE14o-cells) were stimulated by cigarette smoke extract (CSE) in vitro to simulate a smoking environment. On this basis, the mechanism of Allyl isothiocyanate (AITC) administration on the expression of MRP1 in CSE-stimulated 16HBE14o-cells was investigated. The effects of CSE on the viability of 16 HBE14o-cells were investigated by an MTT assay. The changes in the mRNA expression levels of nuclear erythroid factor 2 (Nrf2) and MRP1 were investigated in CSE-stimulated 16HBE14o-cells using western blotting and reverse transcription quantitative PCR (RT-qPCR). Immunofluorescence analysis was used
\end{abstract}

Correspondence to: Professor Dianlei Wang, Anhui Province Key Laboratory of Chinese Medicinal Formula, Anhui University of Chinese Medicine, 350 Longzihu Road, Hefei, Anhui 230012, P.R. China

E-mail: dlwang@ahtcm.edu.cn

Professor Zegeng Li, Department of Respiratory Medicine, The First Affiliated Hospital to Anhui University of Chinese Medicine, 117 Meishan Road, Hefei, Anhui 230031, P.R. China

E-mail: 1i6609@126.com

*Contributed equally

Key words: allyl isothiocyanate, cigarette smoke extract, multidrug resistance-associated protein 1, nuclear erythroid 2-related factor 2, JNK signaling pathway to detect Nrf2 nuclear translocation. Incubation of the cells with 5\% CSE for $24 \mathrm{~h}$ had minor effects on cell viability and resulted in the activation of the JNK and p38MAPK signaling pathways. AITC activated the JNK pathway, inhibited the activation of the p38MAPK pathway in 16HBE14o-cells stimulated by 5\% CSE and upregulated the expression levels of Nrf2 and MRP1 in a time-dependent manner. The upregulation of Nrf2, MRP1 and of Nrf2, and MRP1 mRNA expression levels in CSE-stimulated cells was inhibited by pretreatment with SP600125 (a JNK pathway inhibitor). Furthermore, the fluorescence intensity in the nucleus was significantly enhanced following AITC pretreatment and the analysis indicated nuclear translocation of Nrf2 in the cells. These results indicated that Nrf2 and MRP1 expression levels in CSE-stimulated cells were altered following AITC pretreatment. Thus demonstrating that the primary mechanism may be associated with activation of the JNK pathway, while the p38MAPK pathway may not be involved.

\section{Introduction}

Chronic obstructive pulmonary disease (COPD) is a common disease worldwide, which contributed to $>6 \%$ of the total death rate in 2012 (1). The classic definition of COPD is a preventable and treatable lung disease characterized by airflow limitation caused by abnormal inflammatory response of the lungs to the inhalation of noxious particles or toxic gases (2). Oxidative stress is another important pathophysiological factor for COPD that affects the severity of the disease $(3,4)$. Therefore, oxidative stress may be considered a novel and effective therapeutic target for the treatment of patients with COPD (5).

The multidrug resistance-associated protein 1 (MRP1) belongs to the MRP family of transporter proteins. This protein was initially characterized as a regulator of cellular resistance to chemotherapeutic agents used for the treatment of several solid tumors. However, a previous study have suggested that MRP1 has a role in protecting normal tissues from toxic effects (6). It has been reported that MRP1 expression is higher 
in normal lung tissues and lower in patients with $\operatorname{COPD}(7,8)$. In addition, our previous study demonstrated that decreased MRP1 protein expression was associated with lung function decline in a COPD mouse model (9).

The Huatan Jiangqi formula is a regimen used to treat chronic bronchitis. Additional pharmacological studies conducted on Chinese herbal medicines resulted in the extended use of this regimen for the treatment of COPD (10). Allyl isothiocyanate (AITC) is the main component of the sovereign drug used in the formula and has been used to investigate the prevention and treatment mechanism of COPD. AITC belongs to a group of naturally occurring isothiocyanates (ITCs). ITCs are a type of phytochemicals derived from some dietary glucosinolates and their breakdown products (11). AITC has been demonstrated to affect certain chronic pathological conditions, such as oxidative stress. Several previous studies have focused on the multiple effects of ITCs on activating phase 2 enzymes, antioxidant enzymes and molecular pathways associated with oxidative stress and inflammation $(12,13)$. Benzyl-ITC and phenethyl isothiocyanate have been revealed to play an antitumor effect via regulation of the expression of MRP1 (14). Preliminary studies indicated that AITC increased the expression and activity of MRP1 in 16HBE14o-cells in a concentration-dependent manner via the JNK signaling pathway $(15,16)$.

Nuclear erythroid 2-related factor 2 (Nrf2) is an important transcriptional activator that induces the expression of several detoxification and biotransformation enzymes. A previous study that investigated Nrf2-mediated lung protection suggested that Nrf2 was a valuable therapeutic target for COPD (17). The activation of the Nrf2/antioxidant responsive element pathway is associated with the expression of the upstream kinases, including mitogen-activated protein kinases (MAPKs), protein kinase C, phosphatidylinositol 3-kinase (PI3K) or transmembrane kinase (18). MAPK families, including c-Jun N-terminal kinase (JNK), extracellular signal-regulating kinases (ERKs) and p38MAPK, are key signaling molecules that regulate the expression of target proteins via their response to mitogenic stimulation or environmental stress (19). The MAPK signaling pathway is involved in the activation of fos and jun family genes and their associated gene products (c-Jun, Jun-b, Jun-d, c-Fos, FOSB, Fra-1 and Fra-2), which belong to the AP-1 family members. AP-1 participates in the regulation of several cell-based functions, including cell proliferation, differentiation, transformation, inflammatory response and lung defense (20). p38MAPKs and JNK belong to the MAPK family, which are implicated in the pathogenesis of several inflammatory-driven conditions (such as atherosclerosis and COPD) and regulate cellular behavior in response to oxidative stress (21). A major protective mechanism of these three pathways is to induce the expression of functionally diverse proteins, such as MRP1.

The aim of the present study was to investigate the ability of AITC to induce MRP1 expression in cigarette smoke extract (CSE)-stimulated 16HBE14o-cells in vitro. The results demonstrated that Nrf2 is a key player in the AITC-activated JNK signaling pathway by regulating MRP1 expression. MRP1 could be used as a potential target for the prevention and treatment of COPD.

\section{Materials and methods}

Cell lines. The human bronchial epithelial cell line 16HBE14owas purchased from the Shanghai Fuxiang Biological Technology Co., Ltd.

Reagents. AITC was purchased from the Anhui Haibei Import and Export Co., Ltd. RPMI 1640 medium and fetal bovine serum (FBS) were purchased from Gibco (Thermo Fisher Scientific, Inc.). SB203580 and SP600125 were purchased from Sigma-Aldrich (Merck KGaA). Monoclonal antibodies for p38 (cat. no. sc-7972; 1:500), phosphorylated (p)-p38 (cat. no. sc-7973; 1:500), p-JNK (cat. no. sc-6254; 1:500) and JNK (cat. no. sc-7345; 1:500) were purchased from Santa Cruz Biotechnology, Inc. Anti-MRP1 (cat. no. ab32574; 1:500) and anti-Nrf2 (cat. no. ab137550; 1:500) were purchased from Abcam. The culture plate was purchased from Corning, Inc. The TRIzol ${ }^{\circledR}$ reagent was from Invitrogen (Thermo Fisher Scientific, Inc.). The PCR upstream and downstream primers were synthesized by Shanghai Jieyi Biotechnology Co., Ltd. Anti- $\beta$-actin (cat. no. TA-09; 1:1,000), goat anti-mouse IgG (cat. no. ZB-2305; 1:5,000) and goat anti-rabbit IgG (cat. no. ZB-2301; 1:5,000) were obtained from the Beijing Zhongshan Jinqiao Biological Reagent Company.

Preparation of CSE and AITC liquid. A Huangshan brand cigarette (tar content, $0.8 \mathrm{mg} / \mathrm{one}$ cigarette; nicotine content, $0.8 \mathrm{mg} /$ one cigarette) was continuously inhaled and the cigarette smoke (CS) was collected through a self-made negative pressure device. The smoke was dissolved in $5 \mathrm{ml}$ RPMI-1640 medium. Subsequently, the $\mathrm{pH}$ of the prepared smoke extract solution was adjusted to 7.4 with the addition of $\mathrm{NaOH}$. The samples were filtered and sterilized with a $0.22 \mu \mathrm{m}$ microporous membrane. The concentration of CSE prepared by the aforementioned method was regarded as $100 \%$. In the present study, the RPMI-1640 medium was diluted accordingly and used for the in vitro tests within $30 \mathrm{~min}$. AITC (purity 98\%) was dissolved in a small amount of DMSO and the solution was diluted with RPMI-1640 medium. The stock solution with a concentration of $100 \mu \mathrm{M}$ was prepared, filtered by $0.22 \mu \mathrm{m}$ microporous membrane and preserved at $-20^{\circ} \mathrm{C}$.

Cell culture. The cells $\left(1 \times 10^{4}-10^{5}\right.$ cells $\left./ \mathrm{ml}\right)$ were grown in RPMI-1640 medium supplemented with $10 \%$ FBS and maintained at $37^{\circ} \mathrm{C}$ in a $5 \% \mathrm{CO}_{2}$ atmosphere. Following $24 \mathrm{~h}$ of incubation, the cell growth was observed under an inverted microscope (Nikon eclipse TS100; Nikon Corporation) and the solution was changed (RPMI-1640 with $10 \%$ FBS). The liquid was changed according to the cell growth conditions. The cell cultures were passaged until they reached $80-90 \%$ confluence in the culture bottle. The cells used for the experiments were passaged for a maximum number of 5 times.

Detection of CSE cytotoxicity in 16HBE14o-cells via an MTT assay. 16HBE14o-cells were seeded at a density of $5 \times 10^{4} / \mathrm{ml}$ into the 96-well plate. The experiments included a zero group, a control group and an experimental group. The zero group did not contain cells. The cells were cultured with RPMI-1640 medium containing $10 \%$ FBS. When the cells were cultured to $\sim 70 \%$ confluence, the medium was changed to a fresh 
serum-free RPMI-1640 medium, which was used for $24 \mathrm{~h}$. Following $24 \mathrm{~h}$ of incubation, the solution was changed. The concentration range of CSE used was as follows: 1, 2.5, 5, $10,25,50$ and $100 \%$. The dilutions were performed in fresh serum-free RPMI-1640 medium. A total of $100 \mu \mathrm{l}$ of different concentrations of CSE was added in the experimental groups and maintained at $37^{\circ} \mathrm{C}$ in a $5 \% \mathrm{CO}_{2}$ atmosphere, whereas no CSE was added in the control group. The cell viability was determined following incubation for 12, 24 and $48 \mathrm{~h}$. A total of $20 \mu \mathrm{l} /$ well MTT solution $(5 \mathrm{mg} / \mathrm{ml})$ was added to the culture medium. The culture plate was gently shaken and incubated at a constant temperature incubator (Thermo Fisher Scientific, Inc.) for $3 \mathrm{~h}$. The medium was removed and $150 \mu \mathrm{l}$ DMSO was added to each well. The plate was shaken at low speed for $10 \mathrm{~min}$. The absorbance value of each hole was measured by ELISA microplate reader at $490 \mathrm{~nm}$. The cell survival rate was calculated by the following formula: Cell survival=[(experimental group-zero group $) /($ control group-zero group)] x $100 \%$.

Western blot analysis. The cells were harvested following treatment with CSE, the inhibitor or AITC (The experimental groups and treatments were according to the following steps: 1) CSE stimulation with different durations: Control group was not given any intervention; Experimental groups were treated with 5\% CSE for 5, 15, 30, 60 and $120 \mathrm{~min}$, respectively. 2) CSE stimulation after AITC pretreatment for different time: i) Control group, cells were not given any intervention; ii) $5 \% \mathrm{CSE}$ intervention for $24 \mathrm{~h}$; iii) $40 \mu \mathrm{M}$ AITC pretreatment for $0.5 \mathrm{~h}$ was followed by $5 \%$ CSE intervention for $24 \mathrm{~h}$; iv) $40 \mu \mathrm{M}$ AITC pretreatment for $1 \mathrm{~h}$ was followed by $5 \%$ CSE intervention for $24 \mathrm{~h}$; v) $40 \mu \mathrm{M}$ AITC pretreatment for $2 \mathrm{~h}$ was followed by $5 \% \mathrm{CSE}$ intervention for $24 \mathrm{~h}$; and vi) $40 \mu \mathrm{M}$ AITC pretreatment for $4 \mathrm{~h}$ was followed by $5 \% \mathrm{CSE}$ intervention for $24 \mathrm{~h}$. (3) CSE stimulation after signaling pathway inhibitors pretreatment: i) Control group, cells were not given any intervention; ii) 5\% CSE intervention for $24 \mathrm{~h}$; iii) $10 \mu \mathrm{M} \mathrm{SB} 203580$ (p38 inhibitor; Sigma-Aldrich; Merck KGaA) pretreatment for $1 \mathrm{~h}$ was followed by $5 \%$ CSE intervention for $24 \mathrm{~h}$; and iv) $20 \mu \mathrm{M}$ SP600125 (JNK inhibitor; Sigma-Aldrich; Merck KGaA) pretreatment for $1 \mathrm{~h}$ was followed by $5 \%$ CSE intervention for $24 \mathrm{~h}$. All groups were maintained at $37^{\circ} \mathrm{C}$ in a $5 \% \mathrm{CO}_{2}$ atmosphere.), and gently washed with ice-cold PBS, placed on ice for $10 \mathrm{~min}$ and suspended in $1 \mathrm{X}$ cell lysis buffer, supplemented with $2 \mathrm{mM}$ phenylmethane sulfonyl fluoride and a proteinase inhibitor mixture. All lysates were centrifuged at $4^{\circ} \mathrm{C}$ at $13,000 \mathrm{x} \mathrm{g}$ for $10 \mathrm{~min}$. The protein concentration of the supernatant was measured using BCA reagents. The proteins $(\sim 25 \mu \mathrm{g} /$ lane) were separated via SDS-PAGE on a $6 \%$ gel, and then subsequently transferred to a polyvinylidene difluoride membrane. The membrane was blocked in a buffer containing $5 \%$ non-fat milk, $1 \%$ Tween-20, $1 \mathrm{M}$ Tris- $\mathrm{HCl}$ and $0.5 \mathrm{M} \mathrm{NaCl}$ for $2 \mathrm{~h}$ at room temperature with agitation. The proteins were probed with specific antibodies. The membranes were incubated overnight at $4{ }^{\circ} \mathrm{C}$ with a primary antibody, followed by a secondary antibody for $2 \mathrm{~h}$ at room temperature, and then the bands were visualized using the ECL Plus kit (Pierce; Thermo Fisher Scientifc, Inc.) according to the manufacturer's instructions. The Kodak 1D image analysis software (version 3.5; Shanghai Seebio Biotechnology Co., Ltd.) was used to analyze the western blotting results.

RNA extraction and reverse transcription-quantitative $P C R$ (RT-qPCR). Total RNA was isolated from 16HBE14o-cells using TRIzol ${ }^{\circledR}$ reagent. The experimental protocol was performed following the manufacturer's instructions. The RNA concentration was estimated from the A260/A280 ratio, as determined by measuring the absorbance at 260 and $280 \mathrm{~nm}$. The absorbance measurements were performed using a Hitachi spectrophotometer (model U1100) and the RNA integrity was verified by agarose gel (1.5\%) electrophoresis. cDNA was generated using the High-Capacity cDNA Archive Kit (TransGen Biotech, Inc.) according to the manufacturer's instructions (incubated at $25^{\circ} \mathrm{C}$ for $10 \mathrm{~min}, 42^{\circ} \mathrm{C}$ for $15 \mathrm{~min}$ and inactivated at $85^{\circ} \mathrm{C}$ for $5 \mathrm{sec}$ ). RT-qPCR was performed using the SYBR-Green Master Mix system (Applied Biosystems; Thermo Fisher Scientific, Inc.) on an ABI 7500 real-time PCR machine (Applied Biosystems; Thermo Fisher Scientific, Inc.). Following the reverse transcription reaction, $2 \mu l$ of the resultant cDNA was used for PCR amplification reaction as follows: $10 \mathrm{~min}$ at $95^{\circ} \mathrm{C}$, followed by 40 cycles of $15 \mathrm{sec}$ at $95^{\circ} \mathrm{C}$ and $1 \mathrm{~min}$ at $60^{\circ} \mathrm{C}$. The primer sequences used were as follows: MRP1, forward 5'-CCTGGAGCTGGC CCACCTGA-3' and reverse 5'-CGCTGCCCGACACTGAGG TT-3'; Nrf2, forward 5'-TCCCAGCAGGACATGGATTT-3' and reverse 5'-GCTCATACTCTTTCCGTCGCT-3'. $\beta$-actin was used as the housekeeping gene and the following primers were used: Forward 5'-CAAGGCTGTGGGCAAGGT-3' and reverse 5'-GGAAGGCCATGCCAGTGA-3'. mRNA relative expression was quantifed using the $2^{-\Delta \Delta \mathrm{Cq}}$ method (22).

Immunofluorescence analysis. Treated 16HBE14o-cells were harvested and seeded $\left(\sim 5 \times 10^{4} / \mathrm{ml}\right)$ onto glass coverslips for immunofluorescence analysis. The glass coverslips were washed twice with cold PBS for 5 min and fixed in 4\% paraformaldehyde for $30 \mathrm{~min}$ at room temperature. The resulting glass coverslips were incubated with $0.5 \%$ Triton X-100 for $10 \mathrm{~min}$. Following incubation, the cells were blocked with $5 \%$ bovine serum albumin (Beijing Leagene Biotechnology Co., Ltd.) for $30 \mathrm{~min}$ at room temperature, and incubated overnight with the Nrf2 antibody (1:100) at $4^{\circ} \mathrm{C}$. Following washing with PBS, the cells were stained with FITC-conjugated anti-rabbit IgG antibody (1:100) for $1 \mathrm{~h}$ at room temperature. DAPI was used to re-stain the nuclei for $10 \mathrm{~min}$ at room temperature before washing with PBS. The expression of Nrf2 was assessed by immunofluorescence microscopy (magnification, x400 times; scale bar, $50 \mu \mathrm{m})$.

Statistical analysis. Data are presented as the mean \pm standard deviation. Statistical analyses were performed with SPSS 23.0 (IBM Corp.). One-way ANOVA and Dunnett's multiple-comparisons test and Tukey's post hoc test were used to calculate the differences among experimental groups. $\mathrm{P}<0.05$ was considered to indicate a statistically significant difference.

\section{Results}

Stability investigation of self-made CSE. The absorbance of self-produced CSE was determined by spectrophotometry 


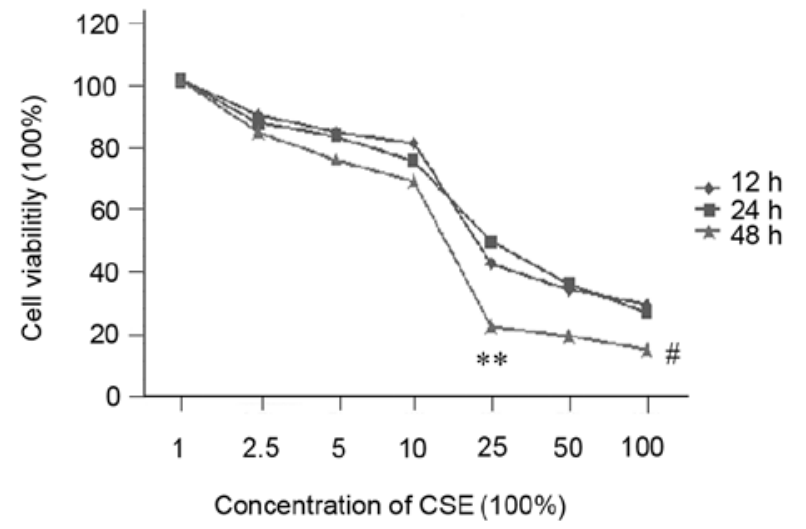

Figure 1. Cytotoxicity of different concentrations of CSE for 12, 24 and $48 \mathrm{~h}$ in human bronchial epithelial cells, as determined by an MTT assay $(n=6)$. ${ }^{* *} \mathrm{P}<0.01$ vs. $10 \% \mathrm{CSE}$; $\mathrm{P}<0.05$ vs. 12 and $24 \mathrm{~h}$ group. CSE, cigarette smoke extract.

at $320 \mathrm{~nm}$. The results indicated no significant differences in the absorbance of self-made CSE derived from different batches $(\mathrm{P}>0.05)$, indicating that the preparation method was stable and reproducible (data not shown).

MTT assay. The cytotoxicity of CSE was determined in 16HBE14o-cells. The number of cells was optimized for the cell proliferation experiments. The inhibitory effect of CSE on 16HBE14o-cells was concentration- and time-dependent (Fig. 1). No significant changes were noted in the cell inhibitory effect following incubation of the cells with 1, 2.5, 5 and $10 \%$ CSE for $48 \mathrm{~h}$. However, the inhibitory rate was significantly increased when the CSE concentration was increased from 10 to $25 \%$. No significant difference was noted in the inhibitory rate between the two types of treatment (50\% CSE and $100 \%$ CSE). The inhibitory rates were similar following incubation for 12 and $24 \mathrm{~h}$ at each concentration of CSE. However, the inhibitory rate following CSE incubation at $48 \mathrm{~h}$ was significantly lower than that of the 12 and $24 \mathrm{~h}$ time periods. Therefore, $5 \%$ CSE was selected as the optimum concentration for subsequent experiments.

Effects of the JNK and p38MAPK signaling pathways in CSE-stimulated $16 \mathrm{HBE} 14 \mathrm{o}$-cells following pretreatment with AITC. Although CSE-induced phosphorylation of the MAPK pathway-associated proteins has been studied in different lung cells $(23,24)$, the effects of CSE on 16HBE14o-cells remains unclear. To explore the upstream signaling pathways responsible for CSE-induced Nrf2 activation and upregulation of MPR1 expression in 16HBE14o-cells, the effects of CSE were examined on the phosphorylation of JNK and p38. The cells were treated with 5\% CSE for 5, 15, 30, 60 and 120 min prior to cell harvest. Subsequently, the phosphorylation of JNK and p38 were examined by western blotting. The expression levels of p-p38 and p-JNK were significantly increased following incubation with 5\% CSE (Fig. 2). This effect was more notable at $5 \mathrm{~min}$. Treatment of the cells with 5\% CSE activated the JNK and p38MAPK signaling pathways. In the present study, the main purpose of the aforementioned experimental design was to verify whether 5\% CSE could activate JNK and p38MAPK signaling pathways, so the incubation time set was relatively short. However, in the follow-up experiments, CSE-stimulated 16HBE140-cells were used to preliminarily verify whether AITC could inhibit the occurrence and development of COPD induced by CSE stimulation. As a long process of CSE stimulation is required to investigate the effect of AITC on 16HBE14o-cells under CSE stimulation, the stimulation time was too short to be meaningful. Combined with the MTT results, cells were treated with 5\% CSE for $24 \mathrm{~h}$ and the survival rate could reach $>80 \%$, which had a small effect on cells and was conducive to the progress of subsequent studies. Thus, 16HBE14o-cells were selected and treated with 5\% CSE for $24 \mathrm{~h}$. Subsequently, the effects of AITC on 16HBE14o-cells were investigated by $5 \%$ CSE stimulation. The cells were treated with AITC ( $40 \mu \mathrm{M})$ for $0.5,1,2$ and $4 \mathrm{~h}$ prior to application of 5\% CSE for $24 \mathrm{~h}$. The ratio of p-JNK/total (t)-JNK increased gradually in the different AITC treatment groups ( 0.5 and $1 \mathrm{~h}, \mathrm{P}<0.05 ; 2$ and $4 \mathrm{~h}, \mathrm{P}<0.01$; Fig. 3 ). It is noteworthy that the detection of $\mathrm{p}-\mathrm{JNK}$ and $\mathrm{JNK}$ proteins resulted in the appearance of two bands. The upper band was the target band. This could be due to the low antibody specificity. In contrast to the increased ratio of the $\mathrm{p}-\mathrm{JNK} / \mathrm{t}-\mathrm{JNK}$, the ratio of p-p38/t-p38 was significantly decreased by AITC pretreatment $(0.5$ and $1 \mathrm{~h}$, $\mathrm{P}<0.05 ; 2$ and $4 \mathrm{~h}, \mathrm{P}<0.01)$. The levels of Nrf2 and MRP1 were significantly higher in $16 \mathrm{HBE} 14 \mathrm{o}$-cells that were stimulated with CSE following AITC pretreatment compared with the single CSE stimulation group $(\mathrm{P}<0.05)$. AITC pretreatment at 1, 2 and $4 \mathrm{~h}$ enhanced the Nrf2 protein expression by 1.74-, 1.76- and 2-fold, whereas the MRP1 protein expression levels were increased by 1.64-, 1.58- and 1.87-fold, respectively.

Effects of JNK and p38MAPK signaling pathway inhibitors on Nrf2 and MRP1 protein levels in 16HBE14o-cells following CSE stimulation. To determine whether the JNK or p38 MAPK signaling pathway is involved in the regulation of $\mathrm{Nrf} 2$ and MRP1 protein expression in CSE-stimulated 16HBE14o-cells, the cells were treated with JNK and p38 MAPK signaling pathway inhibitors. $16 \mathrm{HBE} 14 \mathrm{o}$-cells were treated with two specific signaling pathway inhibitors for $1 \mathrm{~h}$ and subsequently co-incubated with 5\% CSE for $24 \mathrm{~h}$. Specifically, SB203580 $(10 \mu \mathrm{M})$ was used to inhibit the p38MAPK pathway and SP600125 $(20 \mu \mathrm{M})$ was used for the JNK pathway. The data indicated that CSE treatment significantly increased p-p38/t-p38, p-JNK/t-JNK, Nrf2 and MRP1 expression (Fig. 4). Following treatment of $16 \mathrm{HBE} 14 \mathrm{o}-\mathrm{cells}$ with the p38MAPK signaling pathway inhibitor SB203580, the CSE-induced increase in the ratio of p-p38/t-p38 was reversed and the expression levels of p-JNK/t-JNK and MRP1 were slightly decreased compared with those of the CSE treatment group. In addition, the effects of the JNK signaling pathway inhibitor SP600125 on the phosphorylation of JNK, Nrf2 and MRP1 in 16HBE14o-cells were investigated. A slight decrease in the ratio of p-p38/t-p38 compared with that of the CSE treatment group was observed. Whereas, SP600125 decreased JNK phosphorylation by 3.4-fold $(\mathrm{P}<0.01)$, Nrf2 protein expression levels by 1.5 -fold $(\mathrm{P}<0.05)$ and MRP1 protein expression levels by 2.25 -fold compared with those of the CSE stimulation group $(\mathrm{P}<0.01)$.

Effects of JNK and p38MAPK signaling pathway inhibitors on the mRNA levels of Nrf2 and MRPI in 16HBE14o-cells following CSE stimulation. RT-qPCR was used to investigate 
A

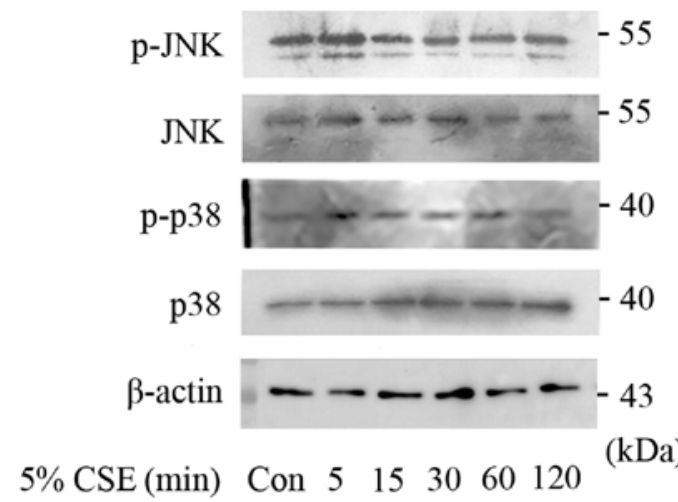

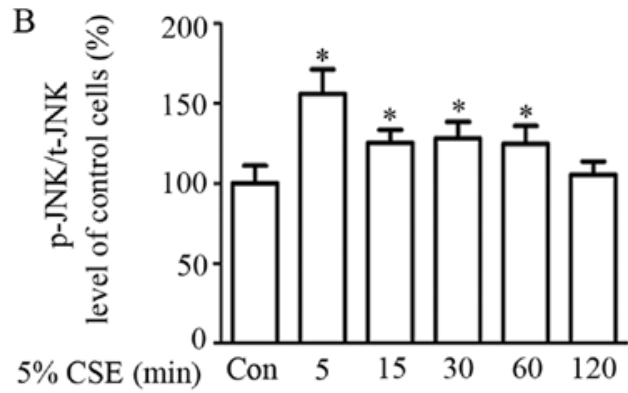

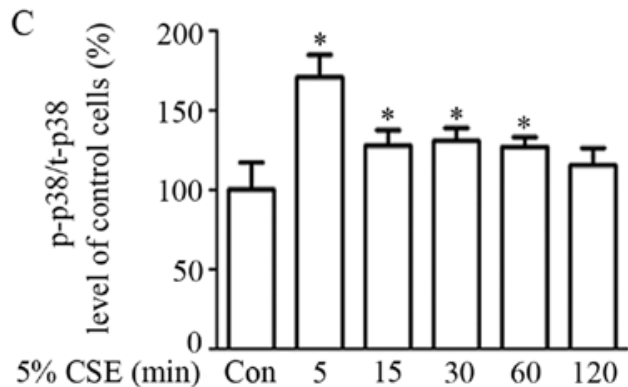

Figure 2. Effect of 5\% CSE on the relative expression of p-JNK, JNK, p-p38 and p38 proteins in human bronchial epithelial cells. (A) Representative western blots and semi-quantification of (B) p-JNK/t-JNK and (C) p-p38/t-p38. ${ }^{*} \mathrm{P}<0.05$ vs. control group. CSE, cigarette smoke extract; JNK, c-Jun N-terminal kinase; p-, phosphorylated; t-, total.

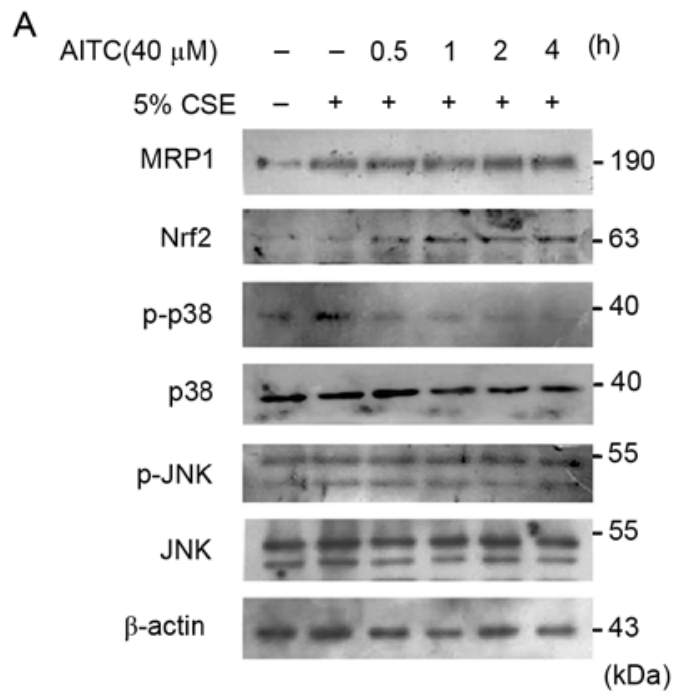

B

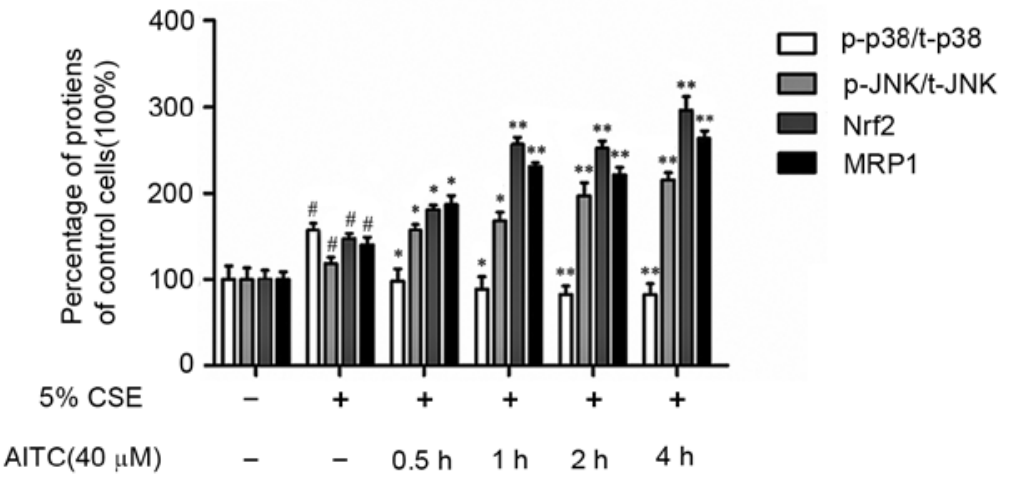

Figure 3. Alteration of Nrf2/MRP1 expression and phosphorylation levels of JNK/p38 with or without CSE in AITC-pretreated cells. (A) Representative western blots and (B) semi-quantification of p-p38/t-p38, p-JNK/t-JNK, Nrf2 and MRP1 protein expression. ${ }^{~} \mathrm{P}<0.05$ vs. control group; ${ }^{*} \mathrm{P}<0.05$ and ${ }^{* * *} \mathrm{P}<0.01$ vs. CSE group. Nrf2, nuclear erythroid factor 2; MRP1, multidrug resistance-associated protein 1; JNK, c-Jun N-terminal kinase; CSE, cigarette smoke extract; AITC, Allyl isothiocyanate; p-, phosphorylated; t-, total.

these two signaling pathways following treatment with inhibitors for $60 \mathrm{~min}$. Incubation of the cells with 5\% CSE for 24 h regulated Nrf2 and MRP1 mRNA expression. 5\% CSE treatment significantly increased Nrf2 and MRP1 mRNA levels compared with those of the control group $(\mathrm{P}<0.01$; Fig. 5). Following treatment of the cells with the p38 MAPK signaling pathway inhibitor SB203580, no significant change was noted in the mRNA levels of Nrf2 and MRP1 compared with those of the control group ( $\mathrm{P}>0.05)$. By contrast, the JNK signaling pathway specific inhibitor SP600125 significantly decreased Nrf2 mRNA $(\mathrm{P}<0.01)$ and MRP1 mRNA $(\mathrm{P}<0.05)$ levels compared with those of the control group. Furthermore, treatment of the cells with SB203580 and CSE in combination did not significantly change the mRNA levels of Nrf2 and MRP1 compared with those of the CSE group ( $\mathrm{P}>0.05)$. Nonetheless, treatment of the cells with SP600125 and CSE significantly decreased the mRNA expression levels of Nrf2 and MRP1 compared with those of the CSE treatment group $(\mathrm{P}<0.05)$.

Immunofluorescence analysis of Nrf2 nuclear translocation in cell groups pretreated with AITC prior to CSE stimulation. $\mathrm{Nrf} 2$ is an important nuclear transcription factor that binds to gene promoters and promotes gene expression $(25,26)$. To 
A

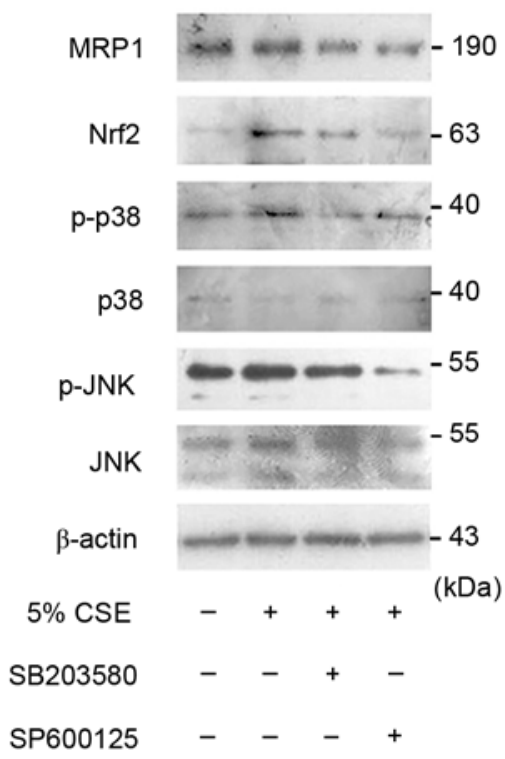

B

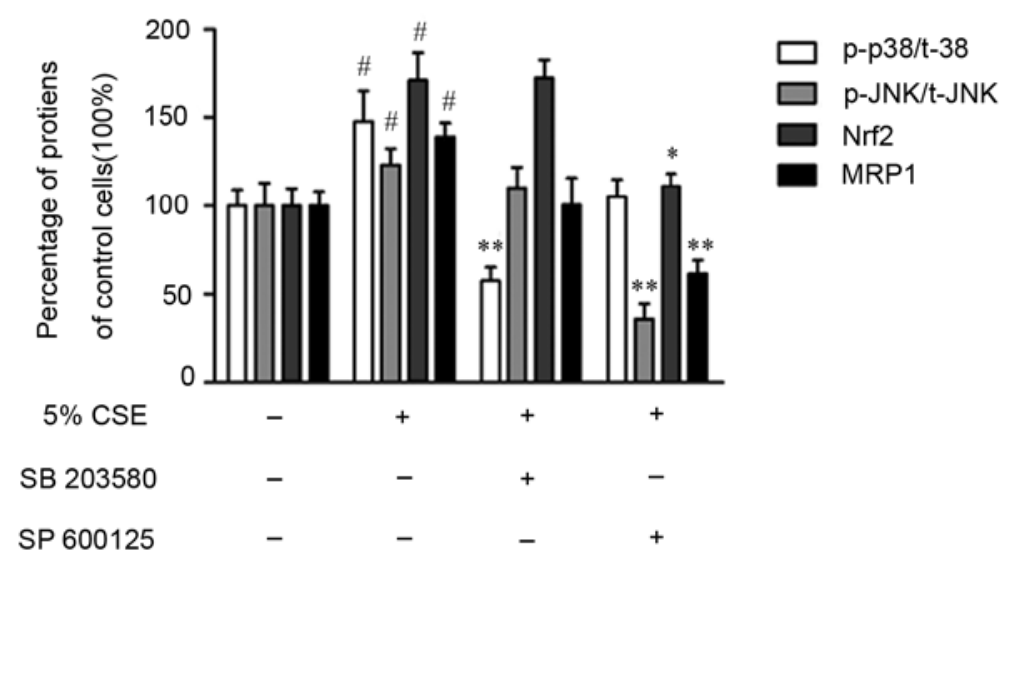

Figure 4. Influence of 5\% CSE with or without JNK/p38MAPK signaling pathway inhibitors on the expression levels of Nrf2/MRP1 and the phosphorylation levels of JNK/p38 proteins in human bronchial epithelial cells. (A) Representative western blots and (B) semi-quantification of p-p38/t-p38, p-JNK/t-JNK, Nrf2 and MRP1. ${ }^{\#} \mathrm{P}<0.05$ vs. control group; ${ }^{*} \mathrm{P}<0.05$ and ${ }^{* *} \mathrm{P}<0.01$ vs. CSE group. CSE, cigarette smoke extract; JNK, c-Jun N-terminal kinase; MAPK, mitogen activated protein kinase; Nrf2, nuclear erythroid factor 2; MRP1, multidrug resistance-associated protein 1; p-, phosphorylated; t-, total.

A

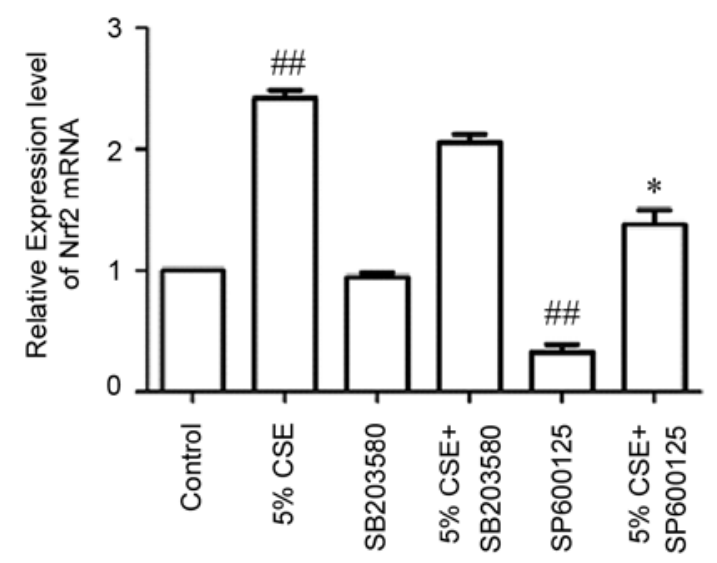

B

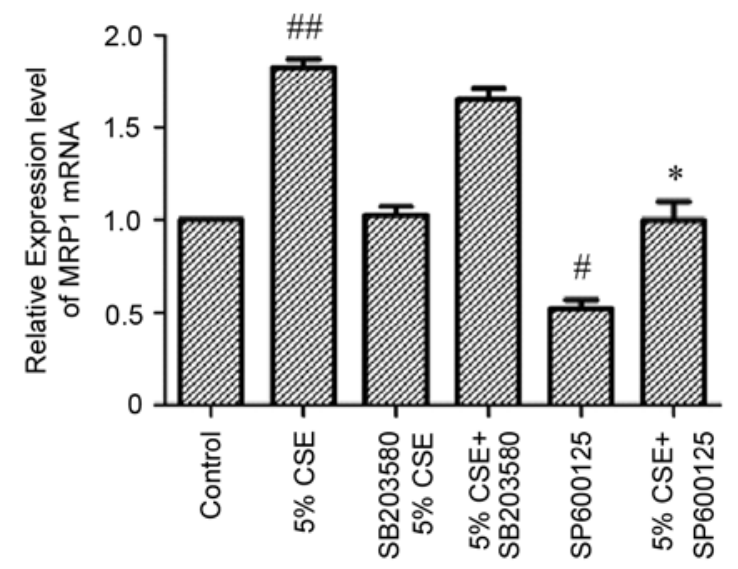

Figure 5. Influence of 5\% CSE with or without c-Jun N-terminal kinase/p38 mitogen activated protein kinase signaling pathway inhibitors on the mRNA expression levels of Nrf2/MRP1 in human bronchial epithelial cells. (A) Representative quantification of (A) Nrf2 and (B) MRP1 mRNA expression. ${ }^{~} \mathrm{P}<0.05$ and ${ }^{\# \#} \mathrm{P}<0.01$ vs. control group; ${ }^{*} \mathrm{P}<0.05$ vs. CSE group. CSE, cigarette smoke extract; Nrf2, nuclear erythroid factor 2; MRP1, multidrug resistance-associated protein 1.

determine the effects of AITC and CSE on Nrf2 activity, the nuclear translocation of $\mathrm{Nrf} 2$ was examined. Under normal physiological conditions Nrf2 was expressed in both the cytoplasm and nucleus in the control group (Fig. 6). CSE treatment increased the fluorescence intensity in the nucleus compared with that of the control group, indicating that it promoted Nrf2 nuclear translocation. However, treatment of the cells with SB203580 for 60 min prior to CSE-induction of Nrf2 nuclear translocation did not lead to any notable differences compared with the control group. Furthermore, the effects of SP600125 treatment on CSE-induced Nrf2 nuclear translocation were investigated. SP600125 pretreatment for 60 min markedly decreased intranuclear fluorescence and increased cytoplasmic fluorescence intensity in CSE-treated cells. In addition, AITC pretreatment for 60 min notably increased intranuclear fluorescence intensity of CSE-treated cells, which suggested that there were increased levels of Nrf2 nuclear translocation.

\section{Discussion}

Mounting evidence suggests that CS notably affects the development and stabilization of COPD $(27,28)$. COPD is caused by cigarette smoking and is primarily due to increased levels of reactive oxygen species contributing to systemic oxidative stress (3). CSE or CS has been reported to increase the ROS-mediated autophagy of airway epithelial cells and to induce apoptosis in a variety of lung cells (29). Moreover, it induces protease/antiproteinase imbalance in lung tissue 


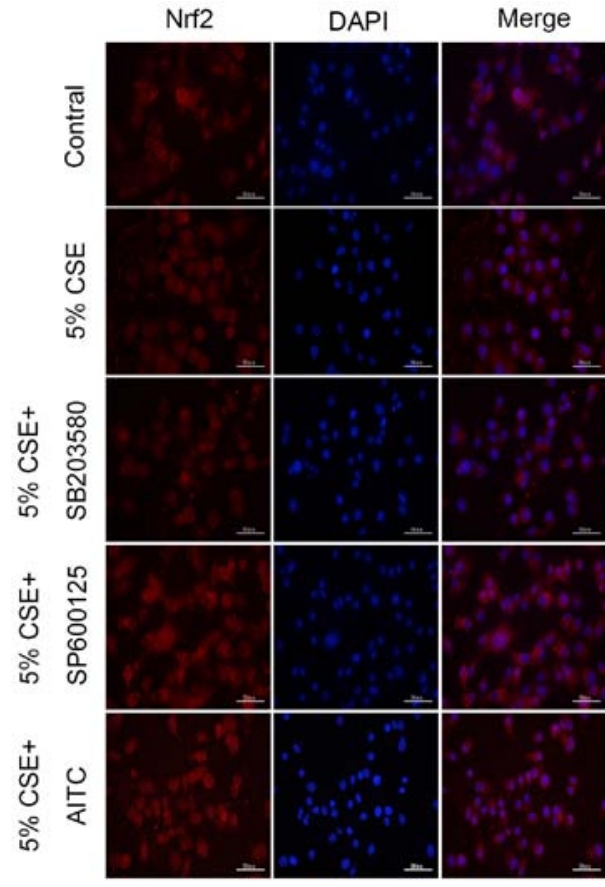

Figure 6. Influence of 5\% CSE with or without Jun N-terminal kinase/p38 mitogen activated protein kinase signaling pathway inhibitors, and AITC treatment. The nuclear translocation of Nrf2 was detected by immunofluorescence staining in human bronchial epithelial cells. CSE, cigarette smoke extract; Nrf2, nuclear erythroid factor 2; AITC, Allyl isothiocyanate.

and promotes the production of additional inflammatory cells $(30,31)$. Based on these studies, CSE was used as an exogenous stimulant to explore the expression of MRP1 and its possible mechanism of action. Under CSE stimulation, the early stages of MRP1 protein and mRNA expression induction in 16HBE14o-cells may be associated with oxidative stress. This result was consistent with a previous study demonstrating that certain toxic heavy metals can induce the expression of MRP1 mRNA in zebrafish (32).

The ability of the JNK and p38MAPK signaling pathways to regulate multidrug resistance-related protein expression has also been reported by previous studies (33-36). In our previous study, a model of COPD was established in rats and the underlying mechanism of COPD was elucidated. During the progression of COPD, the protein levels of MRP1 were gradually reduced in the lung of rats, leading to further deterioration of pulmonary function (37). The understanding of the role of MRP1 is critical for the treatment of COPD. Nrf2 is an important transcription factor that may also affect MRP1 expression. The mechanism required for gene expression involves translocation of the transcription factor to the nucleus and binding to the downstream target gene promoter region in order to promote the expression of each target gene. Therefore, the expression levels of the signaling pathway-associated proteins were examined in the present study to assess the underlying mechanism of MRP1 regulation by AITC following CSE stimulation. To explore the role of AITC on the JNK and p38 MAPK signaling pathways following CSE-induced expression of Nrf2 and MRP1 in 16HBE14o-cells, western blotting and RT-qPCR were used in samples from cell protein and RNA extracts.

CSE has been reported to regulate intracellular signaling pathways $(38,39)$. Previous studies have reported that
CSE exerts different effects on the JNK and MAPK p38 signaling pathway. CSE activated the ERK1/2, p38 and JNK pathways in order to promote the induction of apoptosis in lung fibroblasts (23). In lung epithelial cells, CSE induced lung injury by activating the p38 and JNK pathways (40). CSE is also involved in the apoptosis of retinal pigment epithelial cells by increasing p-p38 protein expression (41). The results of the present study indicated that $\mathrm{p}-\mathrm{JNK} / \mathrm{t}-\mathrm{JNK}$ and p-p38/t-p38 were significantly increased following CSE stimulation for 5-60 min, indicating that CSE activated the JNK and p38 MAPK signaling pathways in 16HBE14o-cells. Concomitantly, the expression levels of Nrf2 and MRP1 mRNA were significantly increased. AITC pretreatment could antagonize the activation of the p38MAPK signaling pathway and upregulate the JNK pathway in a time-dependent manner in 16HBE14o-cells following stimulation with $5 \%$ CSE. AITC further upregulated the expression of Nrf2 and MRP1 in CSE-stimulated 16HBE14o-cells. The results were consistent with the previous study of our research group. Our previous results indicated that AITC upregulated the expression levels of MRP1 in 16HBE cells via the JNK signaling pathway in a concentration-dependent manner $(15,16)$. MRP1 plays a protective role in the COPD process, whereas the JNK pathway also plays an important role in the treatment of COPD (37). The results of this experiment demonstrated that AITC could protect $16 \mathrm{HBE} 140$-cells. In order to clarify the association between the transcription factor Nrf2 and MRP1, a transporter with important cell-protective effects in COPD, the p38MAPK and JNK signaling pathway inhibitors were used in the present study. Following inhibition of the p38 signaling pathway, the expression levels of p-JNK/t-JNK, Nrf2 and MRP1 were slightly decreased, although the results were not significant. The expression levels of Nrf 2 and MRP1 mRNA did not demonstrate a significant change, indicating that the p38MAPK signaling pathway was not involved in the regulation of their expression. Following the inhibition of the JNK signaling pathway, the changes in the ratio of $\mathrm{p}-\mathrm{p} 38 / \mathrm{t}-\mathrm{p} 38$ were not significantly different, whereas the expression levels of Nrf2 and MRP1 were significantly decreased. Concomitantly, the expression levels of Nrf2 and MRP1 mRNA were also significantly decreased, indicating that the JNK pathway was involved in the regulation of Nrf2 and MRP1 expression in CSE-stimulated cells.

Previous studies demonstrated that the activation of MAPKs by plant extracts is associated with the transcriptional activation of Nrf2. However, the specific role of the JNK and p38MAPK signaling pathways in regulating Nrf2 activation varies according to the cell type (42-44). The immunofluorescence results of the present study indicated that Nrf2 was expressed in the cytoplasm and nucleus under normal physiological conditions; a slightly higher cytoplasmic fluorescence intensity was noted. The fluorescence intensity of the CSE group in the nucleus was enhanced, indicating that CSE could promote Nrf2 nuclear translocation compared with the control cells. Pretreatment of the cells with the p38 MAPK signaling pathway specific inhibitor SB203580 for $60 \mathrm{~min}$ did not notably change the fluorescence intensity compared with the CSE group, suggesting that the p38MAPK signaling pathway exhibited no marked effect on Nrf2 nuclear translocation. However, pretreatment of the JNK 
signaling pathway inhibitor SP600125 markedly reduced the fluorescence intensity in the nucleus and enhanced the fluorescence intensity in the cytoplasm, suggesting a blockade of SP600125 on CSE-induced Nrf2 nuclear translocation. Multiple previous studies have demonstrated that the AITC structural analogue sulforaphane is an inducer of the nuclear transcription factor $\operatorname{Nrf} 2(45,46)$. Furthermore, pretreatment of the cells with AITC for 60 min further increased the nuclear fluorescence intensity of CSE-treated cells, indicating that AITC promoted nuclear translocation of Nrf2. Taken together, the present study suggested that inhibition of the JNK signaling pathway reduced the transcriptional activation of Nrf2, whereas blockade of the p38 MAPK signaling pathway did not influence its activation.

In summary, the present study demonstrated that $5 \%$ CSE activated JNK and p38MAPK signaling pathways and upregulated the expression levels of Nrf2 and MRP1 proteins in 16HBE14o-cells. The regulation of AITC on the aforementioned two pathways altered these effects. AITC promoted the expression of $\mathrm{Nrf} 2$ and MRP1 in CSE-stimulated $16 \mathrm{HBE} 14 \mathrm{o}-$-cells in a time-dependent manner, mainly via activation of the JNK pathway. To the best of our knowledge, the present study indicated for the first time that AITC promoted Nrf2 nuclear translocation in CSE-stimulated 16HBE14o-cells via the JNK signaling pathway, and that the JNK signaling pathway serves an important role in Nrf2 and MRP1 expression induced by AITC in 16HBE14o-cells. These conclusions indicate that AITC has potential applications in the treatment of COPD.

\section{Acknowledgements}

The authors would like to thank Dr Xiaobing Zhang (Department of Psychology, Florida State University) for language editing the manuscript.

\section{Funding}

This study was funded by the National Natural Science Foundation (grant nos. 81473536 and 81001592).

\section{Availability of data and materials}

All data generated or analyzed during this study are included in this published article.

\section{Authors' contributions}

DW and ZL conceived and designed the experiments. MZ, SW and XW performed the experiments. XX, ZY, WF, JW and QW were responsible for data analysis and interpretation. MZ, SW and DW wrote the manuscript. XW and JW confirm the authenticity of all the raw data. MZ and SW contributed equally to this study, and should be regarded as co-first authors. All authors reviewed and approved the final manuscript.

\section{Ethics approval and consent to participate}

Not applicable.

\section{Patient consent for publication}

Not applicable.

\section{Competing interests}

The authors declare that they have no competing interests.

\section{References}

1. World Health Organization: Chronic obstructive pulmonary disease (COPD) Fact sheet March 2015. WHO. http://www.who. int/mediacentre/factsheets/fs315/en/. 2015.

2. Milara J, Peiró T, Serrano A, Guijarro R, Zaragozá C, Tenor H and Cortijo J: Roflumilast N-oxide inhibits bronchial epithelial to mesenchymal transition induced by cigarette smoke in smokers with COPD. Pulm Pharmacol Ther 28: 138-148, 2014.

3. Chung KF and Marwick JA: Molecular mechanisms of oxidative stress in airways and lungs with reference to asthma and chronic obstructive pulmonary disease. Ann NY Acad Sci 1203: 85-91, 2010.

4. MacNee W: Pulmonary and systemic oxidant/antioxidant imbalance in chronic obstructive pulmonary disease. Proc Am Thorac Soc 2: 50-60, 2005.

5. Burgel PR and Martin C: Mucus hypersecretion in COPD: Should we only rely on symptoms?. Eur Respir Rev 19: 94-96, 2010.

6. Cole SP: Targeting multidrug resistance protein 1 (MRP1, ABCC1): Past, present, and future. Annu Rev Pharmacol Toxicol 54: 95-117, 2014.

7. Cole SP, Bhardwaj G, Gerlach JH, Mackie JE, Grant CE, Almquist KC, Stewart AJ, Kurz EU, Duncan AM and Deeley RG: Overexpression of a transporter gene in a multidrug-resistant human lung cancer cell line. Science 258: 1650-1654, 1992.

8. Budulac SE, Postma DS, Hiemstra PS, Lapperre TS, Kunz LI, Vonk JM, Marike Boezen H and Timens W; Glucold Study Group: Multidrug resistance-associated protein 1 and lung function decline with or without long-term corticosteroids treatment in COPD. Eur J Pharmacol 696: 136-142, 2012.

9. Wang DL, Zhang X and Tao XH: Effects of huatan jianqi capsule on the levels of multidrug resistance-associated protein 1 in the bronchial epithelial cells of model rats with chronic obstructive pulmonary disease. Zhongguo Zhong Xi Yi Jie He Za Zhi 32: 955-959, 2012 (In Chinese).

10. Wang GZ, Peng B, Han MX, Li ZG, Han NL and Zhang NZ: The effect of huatan jiangqi capsule on pulmonary function and arterial blood gas in patients with COPD. Zhongguo Zhong Yi Ji Zheng 15: 1311-1312, 2006.

11. Kushad MM, Brown AF, Kurilich AC, Juvik JA, Klein BP, Wallig MA and Jeffery EH: Variation of glucosinolates in vegetable crops of Brassica oleracea. J Agric Food Chem 47: 1541-1548, 1999.

12. Giacoppo S, Galuppo M, Montaut S, Iori R, Rollin P, Bramanti P and Mazzon E: An overview on neuroprotective effects of isothiocyanates for the treatment of neurodegenerative diseases. Fitoterapia 106: 12-21, 2015.

13. Wu X, Zhou $\mathrm{QH}$ and $\mathrm{Xu} \mathrm{K}$ : Are isothiocyanates potential anti-cancer drugs?. Acta Pharmacol Sin 30: 501-512, 2009.

14. Hu K and Morris ME: Effects of benzyl-, phenethyl-, and alpha-naphthyl isothiocyanates on P-glycoprotein- and MRP1-mediated transport. J Pharm Sci 93: 1901-1911, 2004.

15. Wang DL, Wang CY, Cao Y, Zhang X, Tao XH, Yang LL, Chen JP, Wang SS and Li ZG: Allyl isothiocyanate increases MRP1 function and expression in a human bronchial epithelial cell line. Oxid Med Cell Longev 2014: 547379, 2014.

16. Wang SJ, Wang SS, Wang CY, Chen YJ, Li J, Wang XQ, Wang DL, Li ZG, Peng ZL and Fan L: Upregulation of multidrug resistance-associated protein 1 by allyl isothiocyanate in human bronchial epithelial cell: Involvement of c-Jun N-terminal kinase signaling pathway. Oxid Med Cell Longev 2015: 903782, 2015.

17. Boutten A, Goven D, Artaud-Macari E, Boczkowski J and Bonay M: NRF2 targeting: A promising therapeutic strategy in chronic obstructive pulmonary disease. Trends Mol Med 17: 363-371, 2011.

18. Jeong WS, Jun M and Kong AN: Nrf2: A potential molecular target for cancer chemoprevention by natural compounds. Antioxid Redox Signal 8: 99-106, 2006. 
19. Kong AN, Owuor E, Yu R, Hebbar V, Chen C, Hu R and Mandlekar S: Induction of xenobiotic enzymes by the MAP kinase pathway and the antioxidant or electrophile response element (ARE/EpRE). Drug Metab Rev 33: 255-271, 2001.

20. Shaulian E and Karin M: AP-1 in cell proliferation and survival. Oncogene 20: 2390-2400, 2001.

21. Fisk M, Gajendragadkar PR, Mäki-Petäjä KM, Wilkinson IB and Cheriyan J: Therapeutic potential of p38 MAP kinase inhibition in the management of cardiovascular disease. Am J Cardiovasc Drugs 14: 155-165, 2014.

22. Livak KJ and Schmittgen TD: Analysis of relative gene expression data using real-time quantitative PCR and the 2(-Delta Delta $\mathrm{C}(\mathrm{T})$ ) method. Methods 25: 402-408, 2001.

23. Lee H, Park JR, Kim EJ, Kim WJ, Hong SH, Park SM and Yang SR: Cigarette smoke-mediated oxidative stress induces apoptosis via the MAPKs/STAT1 pathway in mouse lung fibroblasts. Toxicol Lett 240: 140-148, 2016.

24. Shang J, Zhao J, Wu X, Xu Y, Xie J and Zhao J: Interleukin-33 promotes inflammatory cytokine production in chronic airway inflammation. Biochem Cell Biol 93: 359-366, 2015.

25. Saetta M, Turato G, Baraldo S, Zanin A, Braccioni F, Mapp CE, Maestrelli P, Cavallesco G, Papi A and Fabbri LM: Goblet cell hyperplasia and epithelial inflammation in peripheral airways of smokers with both symptoms of chronic bronchitis and chronic airflow limitation. Am J Respir Crit Care Med 161: 1016-1021, 2000.

26. Global Initiative for Chronic Obstructive Lung Disease (GOLD): Global Strategy for Diagnosis Management and Prevention of COPD EB/OL. http://www.goldcopd.org/. 2013.

27. Muro S: Cigarette smoking is the most important causal factor for developing chronic obstructive pulmonary disease (COPD). Nihon Rinsho 69: 1735-1740, 2011 (In Japanese).

28. Caramori G, Kirkham P, Barczyk A, Stefano AD and Adcock I Molecular pathogenesis of cigarette smoking-induced stable COPD. Ann N Y Acad Sci Mar 1340: 55-64, 2015.

29. Hwang JW, Chung S, Sundar IK, Yao H, Arunachalam G McBurney MW and Rahman I: Cigarette smoke-induced autophagy is regulated by SIRT1-PARP-1-dependent mechanism: Implication in pathogenesis of COPD. Arch Biochem 500 203-209, 2010.

30. Mercado N, Ito K and Barnes PJ: Accelerated ageing of the lung in COPD: New concepts. Thorax 70: 482-489, 2015.

31. Malhotra D, Thimmulappa R, Vij N, Navas-Acien A, Sussan T, Merali S, Zhang L, Kelsen SG, Myers A, Wise R, et al: Heightened endoplasmic reticulum stress in the lungs of patients with chronic obstructive pulmonary disease: The role of Nrf2-regulated proteasomal activity. Am J Respir Crit Care Med 180: 1196-1207, 2009.

32. Long Y, Li Q, Wang Y and Cui Z: MRP proteins as potential mediators of heavy metal resistance in zebrafish cells. Comp Biochem Physiol C Toxicol Pharmacol 153: 310-317, 2011.

33. Guan J, Chen XP, Zhu H, Luo SF, Cao B and Ding L: Involvement of extracellular signal-regulated kinase/mitogen-activated protein kinase pathway in multidrug resistance induced by $\mathrm{HBx}$ in hepatoma cell line. World J Gastroenterol 10: 3522-3527, 2004.

34. Hayashi K, Pu H, Andras IE, Eum SY, Yamauchi A, Hennig B and Toborek M: HIV-TAT protein upregulates expression of multidrug resistance protein 1 in the blood-brain barrier. J Cereb Blood Flow Metab 26: 1052-1065, 2006.
35. Ronaldson PT, Ashraf T and Bendayan R: Regulation of multidrug resistance protein 1 by tumor necrosis factor alpha in cultured glial cells: Involvement of nuclear factor-kappaB and c-Jun N-terminal kinase signaling pathways. Mol Pharmacol 77: 644-659, 2010.

36. Kwon YS, Chun SY, Nam KS and Kim S: Lapatinib sensitizes quiescent MDA-MB-231 breast cancer cells to doxorubicin by inhibiting the expression of multidrug resistance-associated protein-1. Oncol Rep 34: 884-890, 2015.

37. Wang DL, Zhang X, Tao XH, Cao Y and Peng B: Expression of multidrug resistance-associated protein 1 in bronchial epithelium of model srat with chronic obstructive pulmonary disease and the reversal effects of Huatanjiangqi Capsule on it. Chin J Clin Pharm Therap 17: 621-625, 2012.

38. Shen HJ, Sun YH, Zhang SJ, Jiang JX, Dong XW, Jia YL, Shen J, Guan Y, Zhang LH, Li FF, et al: Cigarette smoke-induced alveolar epithelial-mesenchymal transition is mediated by Rac1 activation. Biochim Biophys Acta 1840: 1838-1849, 2014.

39. Tharakan A, Halderman AA, Lane AP, Biswal S and Ramanathan M Jr: Reversal of cigarette smoke extract-induced sinonasal epithelial cell barrier dysfunction through Nrf2 activation. Int Forum Allergy Rhino 6: 1145-1150, 2016.

40. Yu G, Zeng X, Wang H, Hou Q, Tan C, Xu Q and Wang $\mathrm{H}$ : 14,15-epoxyeicosatrienoic Acid suppresses cigarette smoke extract-induced apoptosis in lung epithelial cells by inhibiting endoplasmic reticulum stress. Cell Physiol Biochem 36: 474-486, 2015.

41. Huang C, Wang JJ, Ma JH, Jin C, Yu Q and Zhang SX: Activation of the UPR protects against cigarette smoke-induced RPE apoptosis through up-regulation of Nrf2. J Biol Chem 290: 5367-5380, 2015.

42. Truong VL, Bak MJ, Jun M, Kong AN, Ho CT and Jeong WS: Antioxidant defense and hepatoprotection by procyanidins from almond (Prunus amygdalus) skins. J Agric Food Chem 62: 8668-8678, 2014

43. Lee DS, Li B, Kim KS, Jeong GS, Kim EC and Kim YC: Butein protects human dental pulp cells from hydrogen peroxide-induced oxidative toxicity via Nrf2 pathway-dependent heme oxygenase-1 expressions. Toxicol In Vitro 27: 874-881, 2013.

44. Ji LL, Sheng YC, Zheng ZY, Shi L and Wang ZT: The involvement of p62-Keap1-Nrf2 antioxidative signaling pathway and JNK in the protection of natural flavonoid quercetin against hepatotoxicity. Free Radic Biol Med 85: 12-23, 2015.

45. Juge N, Mithen RF and Traka M: Molecular basis for chemoprevention by sulforaphane: A comprehensive review. Cell Mol Life Sci 64: 1105-1127, 2007.

46. Heiss E, Herhaus C, Klimo K, Bartsch H and Gerhäuser C: Nuclear factor kappa B is a molecular target for sulforaphane-mediated anti-inflammatory mechanisms. J Biol Chem 276: 32008-32015, 2001.

This work is licensed under a Creative Commons Attribution-NonCommercial-NoDerivatives 4.0 International (CC BY-NC-ND 4.0) License. 